\title{
Constant Flow Control of the Parallel Precision Piston Pump
}

\author{
Tong ZHANG ${ }^{1, a}$, Mingfu $\mathrm{YIN}^{2, \mathrm{~b}}$, * \\ ${ }^{1}$ School of mechanical engineering, Tianjin Polytechnic University, Tianjin, 300387, China \\ ${ }^{2}$ School of mechanical engineering, Tianjin Polytechnic University, Tianjin, 300387, China

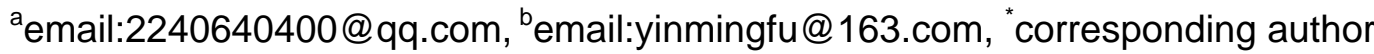

Keywords: Piston Pump, Harmonic Gear Reducer, Constant Flow, MSP430

\begin{abstract}
In order to change the present situation that the traditional axial piston pump can only change flow by controlling the swashplate angle, this paper designs a precision piston pump, introduces in detail its structure and working principle, and uses harmonic gear reducer to improve transmission accuracy and load capacity, and to reduce volume and weight of the system. On this basis, the paper uses the system of parallel piston pump to achieve constant flow output, gives the movement and flow characteristic of double pump, and designs overall scheme and MSP430 MCU hardware system of the control system.
\end{abstract}

\section{Introduction}

The piston pump is widely used in the fields of petroleum, chemical, pharmaceutical etc. It has many advantages, such as higher pressure, compact structure, higher efficiency and convenient flow adjustment, especially in the occasions of high pressure and flow adjustment, and has distinct advantages compared to various types of pumps [1].Though swashplate axial piston pump is often used and its flow is controlled by changing the swashplate angle, the variable mechanism of the swashplate increases volume and weight of the piston pump, and its structure is more complex [2]. Therefore, this paper proposes a precision piston pump, its flow is controlled by speed control of a AC servo motor, completely eliminating the variable mechanism of swashplate and the use of double pump can achieve constant flow output, which reflects the development direction of miniaturization and digital of the hydraulic components.

\section{The Working Principle of the Parallel Precision Piston Pump}

Compared with conventional precision piston pump, driver of the pump that this paper designs is a AC servo motor instead of a stepper motor. AC servo motor has many greater advantages, including control precision, low-frequency characteristics, torque-frequency characteristic, overload capacity and speed response etc[3]. In addition, harmonic gear reducer is used to transmit coaxially rotation of the servo motor. Compared to other reducer, harmonic gear reducer has many advantages, such as larger transmission ratio, higher transmission efficiency, higher load capacity, smaller backlash backlash, more stable transmission, smaller size and weight, and lower noise [4].Thus, it is widely used in the field for precise positioning, including robotics, radar, etc.

The parallel operation system is composed of two sets of the precision piston pump. The output pressure of each piston pump is controlled by a pressure transmitter, and the output flow is controlled by the servo motor driving ball screw to control movement speed of the piston. By controlling two servo motors to coordinate and compensate mutually in the motion process, the system can not only automatically and continuously run for liquid, but also allow liquid to achieve stable flow output at a constant rate. Parallel system components of the precision piston pump is shown in Fig. 1. 


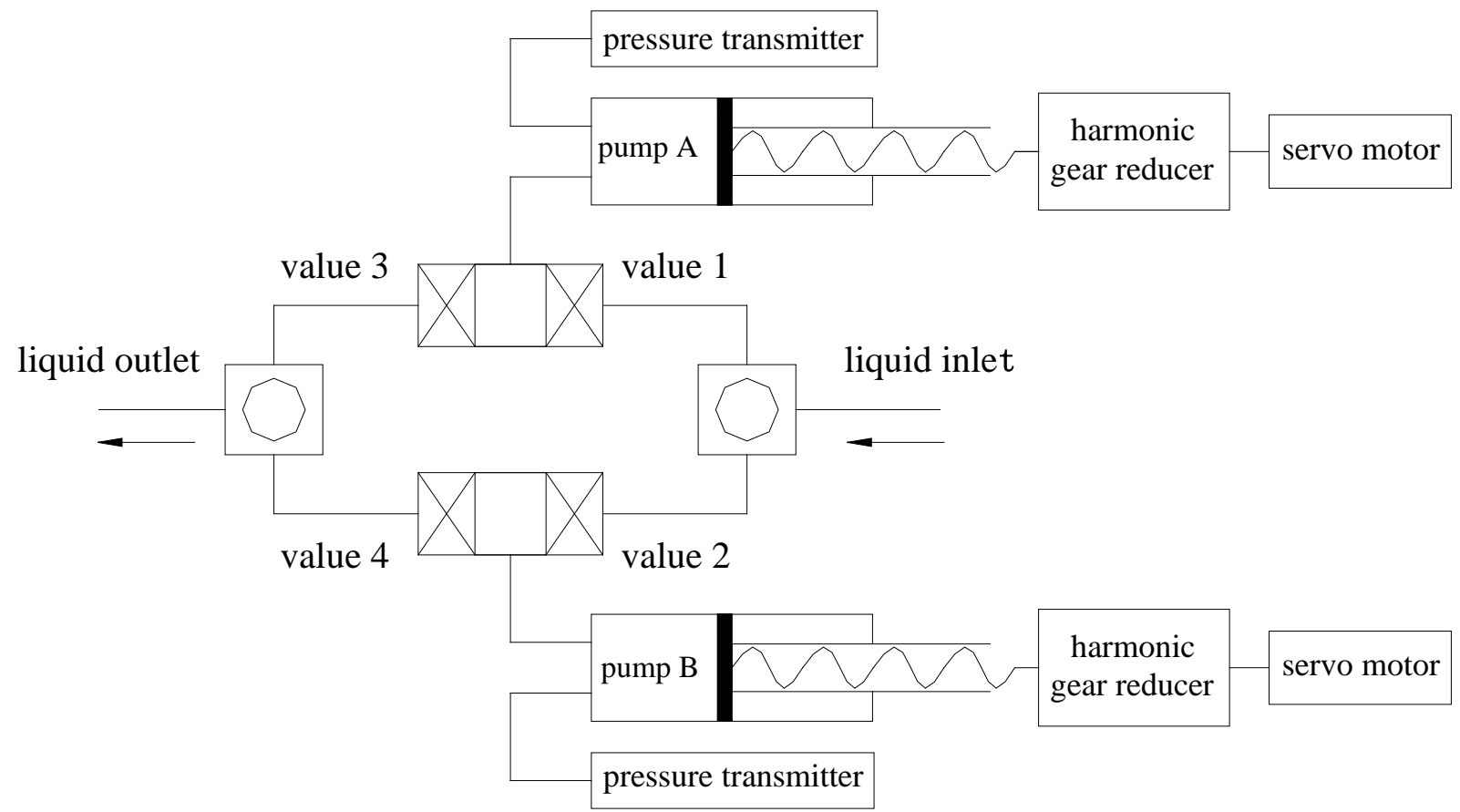

Fig.1. Parallel system components of the precision piston pump

When pump A discharges liquid, pump B rapidly aspirates liquid. And the status of each valve is: valve 2 and 3 are open; valve 1 and 4 are closed. Pump B has been back to the limit position of the aspiration liquid before pump A starts uniform deceleration near limit position to discharge liquid, and control system closes valve 2 and 4. With pressure transmitter of pump A tracking working pressure of the system, control system pressurizes pump B in advance through pressure transmitter of pump B. The value of the pressurization is working pressure tracked. When pump A starts uniform deceleration at acceleration $\mathrm{a}_{1}$, control system opens valve 4 and pump $\mathrm{B}$ also starts uniform acceleration to discharge liquid at acceleration $\mathrm{a}_{1}$.Pump B uniformly accelerates to the steady speed value and control system closes valve 3, meanwhile pump A slows down to 0, which completes alternation. After that, pump B continues to drain, control system opens valve 1 and closes valve 3,and pump A is quickly filled with liquid and pre-pressurized for starting next cycle of the operation. Fig. 2 is the characteristic of double pump movement and flow .

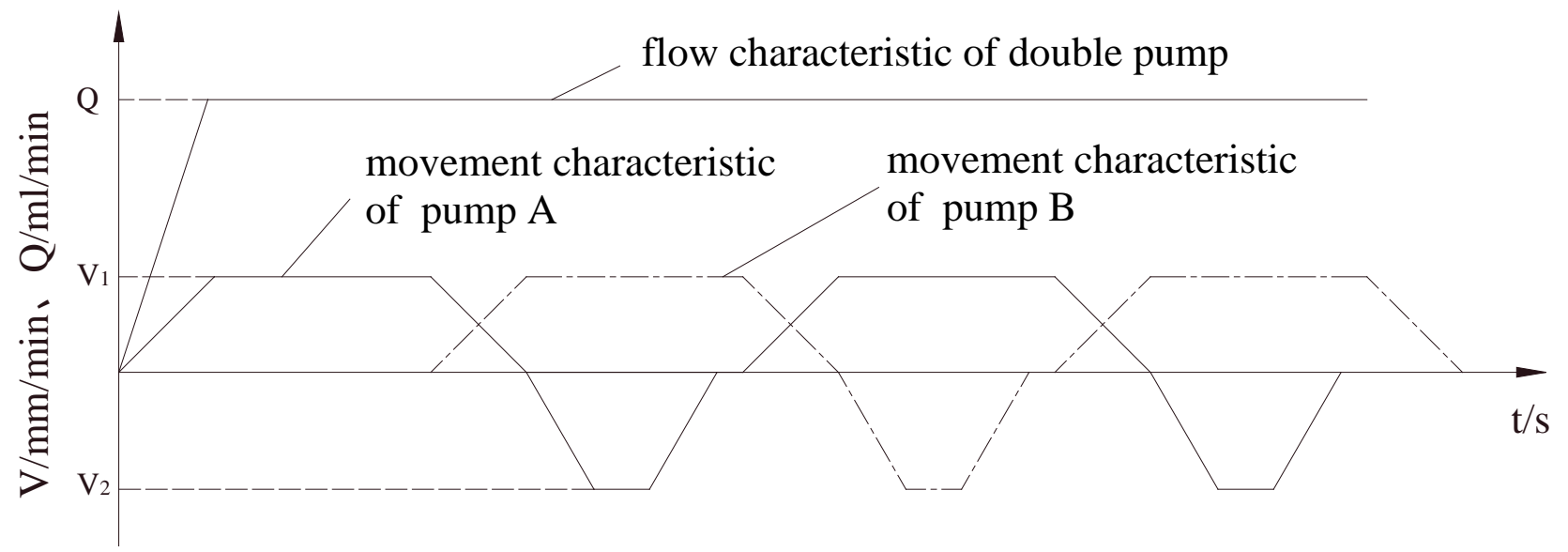

Fig.2. The characteristic of double pump movement and flow

\section{The Design of the Overall Scheme}

Microcontroller is widely used in the control of servo motor, because of its good economy, high reliability, strong processing ability, low voltage and power consumption, simple system, and convenient modularization and use [5].Because the composition of servo system of pump A is the same as pump B, the following description shows only the system of pump A. 
This system adopts a three-phase AC servo motor of Panasonic MINAS A5 series that are high inertia, small capacity and built-in 20 incremental photoelectric encoder, and selects its servo driver supported. PC sends flow instruction signals to the microcontroller, then the microcontroller converts the signals into speed analog voltage and outputs them. According to the voltage signal, the servo motor driver achieves speed control of the servo motor. At the same time, photoelectric encoder measures speed of the servo motor and feedbacks it to the servo driver that will compare the feedback value with target value to obtain output to adjust motor speed, so as to realize closed-loop control of the motor speed. The block diagram of the control system of the parallel precision piston pump is shown in Fig. 3.

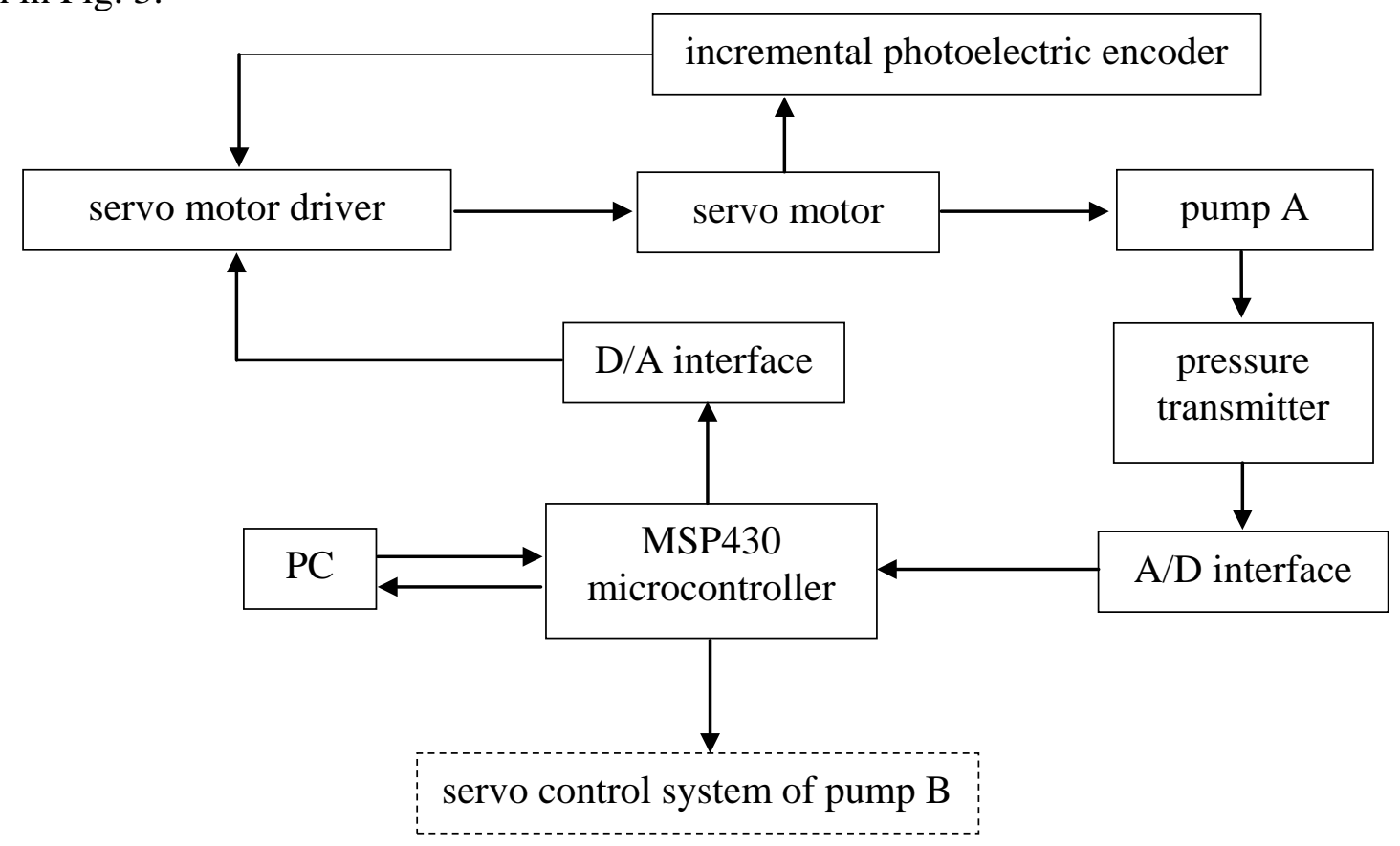

Fig.3. The block diagram of the control system of the parallel precision piston pump

In the flow control system of the parallel piston pump, the PC sends instructions that includes discharging ,aspirating, pre-pressurization, stopping and parameter setting to the microcontroller, and displays pressure of the pump A and B in real time.In figure 4,two pressure transmitters monitor the pressure of pump A and B.When the pump A needs for pre-pressurization, MSP430 microcontroller reads the pressure value of pump $A$ and $B$ to obtain the difference in real time, and the speed command is obtained by adopting PID control algorithm and is sent to the servo motor driver, so as to change pressure of pump A through controlling the servo motor to drive the piston to move.Meanwhile, the pressure value of two pumps is transmitted in real time to the PC.The cycle of feedback control continues and pre-pressurization process is not completed until the pressure of pump A is the same as Pump B.

\section{The Hardware Design of the Microcomputer Controller}

The selection of the microcomputer controller is MSP430F149 of MSP430 series, which has three main functions: The first one is to communicate with PC via RS232 serial port for receiving command signals of the flow and pressure and feed backing the pressure value of pump A and B in real time; The second one is to communicate with pressure transmitter via RS485 serial port for reading pressure value of the pressure transmitter; The third one is to get speed command through the PID control algorithm, and this speed signal is converted into speed analog voltage required for servo driver by DAC interface. MSP430F149 has two USART communication ports that its functions are exactly same and can be used separately to serial communication for RS232 and RS485. So we can use USART0 and USART1 to provide asynchronous communication function. The one is used to achieve RS232 serial communication with PC through the chip MAX3232; the other one is used to achieve RS485 serial communication with pressure transmitter through the chip MAX3078. 
Though MSP430F149 microcontroller has a 12 bit DAC conversion module, we replace it with the chip MAX5442 with a 16 bit conversion precision and faster speed in order to improve the conversion precision[6]. Because USART0 and USART1 serial communication modules have been used for RS232 and RS485 serial communication, the microcontroller controls the chip MAX5442 in analog I/O manner via I/O pins P2.7, P3.1 and P3.3. The digital output signal of I/O is $0 \sim 3.3 \mathrm{~V}$ [7]. The microcontroller outputs analog voltage signal $(-5 \mathrm{~V} \sim+5 \mathrm{~V})$ to the servo driver through D/A interface and low-pass filter.

\section{Conclusion}

The application of harmonic gear reducer and AC servo motor makes constant flow output of the precision piston pump more accurate. The precision piston pump proposed completely eliminates the variable mechanism of swashplate and the use of double pump can achieve constant flow output, which reflects the development direction of miniaturization and digital of the hydraulic components.

\section{References}

[1] Xiaolan Dai. Innovative Design of A NC Bouble-stage Constant Flux Piston Pump[J]. Mechanical Engineering \& Automation,2012(01):100-101.

[2] Fang Lu, Huaichao Wu. Design of the Axial Displacement Piston Pump with Stepper Motor and Its Flow Control[J]. Machine Tool \& Hydraulics, 2013, 41(9):98-100.

[3] Penghui Si. The Comprehensive Comparison of Performance of Stepping Motor and AC Servo Motor. Electronic Test[J]. 2015(13):85-86.

[4] Qingzhu Wan, Zhigang Lu, Ke Wang, Xuzhu Dong, Weiguo Duan, Qingbin Tong, Guibin Li. Precision Harmonic Gear Reducer Transmission Error Analysis[J].Instrument Technique and Sensor,2013(05):51-54.

[5] Yong Sang, Fengtao Lu, Yuebang Dai, Fuhai Duan, Yajie Wang.The STM32 SCM Control System Design onServo Motor Oriented[J]. Instrument Technique and Sensor,2013(05):51-54.

[6] Kaifang $\mathrm{Xu}$, Zhitao Cai. Design of Smart Digital Multifunction Meter Based on MSP430[J].Mechanical \& Electrical Engineering Technology, 2014(06):86-89.

[7] Caiju Li. Research on Servo-Motor Control System Based on SCM of MSP430[J].Mechanical \& Electrical Engineering Technology, 2015,44(11):77-80. 